\title{
Erratum: CozE is a member of the MreCD complex that directs cell elongation in Streptococcus pneumoniae
}

Andrew K. Fenton, Lamya El Mortaji, Derek T. C. Lau, David Z. Rudner and Thomas G. Bernhardt

Nature Microbiology 2, 16237 (2016); published online 12 December 2016; corrected 23 January 2017

In the original version of this Letter, co-author Lamya El Mortaji's name was coded wrongly resulting in it being incorrect when exported to citation databases. This has now been corrected, though no visible changes will be apparent. 